\title{
Plant Disease Detection System for Smart Agriculture
}

\author{
R. Indhu' and K.Thilagavathi \\ ${ }^{1} P G$ student, Department of ECE, Kumaraguru College of Technology, Coimbatore, India \\ ${ }^{2}$ Asst Professor, Department of ECE, Kumaraguru College of Technology, Coimbatore, India
}

\section{ABSTRACT}

Indian economy relies on agriculture to a greater extent. In traditional agriculture, the farmers identify the crop diseases with the help of an expert either by direct visual inspection or by sending the diseased images to experts through online services. Also, continuous monitoring cannot be done manually. The main objective is to develop an android application which identifies and classifies three major diseases - Black horse riding, Brown spot and Bacterial leaf steak. In the plant disease detection system, image to be tested is acquired, pre-processed, segmented and classified based on the disease type. The classification is performed using probabilistic linear classifier called Naive Bayes. The application is developed using Android Studio and the programming language used for the development is java. This application identifies the plant disease based on pixel intensities, predicts the plant growth and sunlight condition if it is good or not. It suggests suitable fertilizers and pesticides depending on the disease type. The average accuracy of the developed application is about $80 \%$ and the implementation of this system reduces manpower and increases productivity.

\section{KEY WORDS: ANDROID APPLICATION, IMAGE PROCESSING, NAIVE BAYES CLASSIFIER, PLANT DISEASES.}

\section{INTRODUCTION}

Human survival is greatly influenced by the food supply from nature, which purely depends upon agriculture. Since the population increases day-by-day, the demand for food also increases. Hence, agriculture is a field that never ends at all. Crop diseases leads to less productivity which in turn affects the crop yield. Crop yield can be increased by detecting the diseases in the early stages. The traditional method of disease detection and classification involves visual inspection, which is expensive, time consuming and sometimes provides incorrect results. Hence digital image processing techniques and probabilistic neural network algorithm-Naive Bayes are applied to improve the accuracy of detecting and classifying the crop diseases

\section{ARTICLE INFORMATION}

${ }^{*}$ Corresponding Author: indhu.18mco@kct.ac.in

Received 15th Oct 2020 Accepted after revision 5th Dec 2020

Print ISSN: 0974-6455 Online ISSN: 2321-4007 CODEN: BBRCBA

Thomson Reuters ISI Web of Science Clarivate Analytics USA and Crossref Indexed Journal

\section{Clarivate
Analytics}

NAAS Journal Score 2020 (4.31) SJIF: 2020 (7.728)

A Society of Science and Nature Publication,

Bhopal India 2020. All rights reserved.

Online Contents Available at: http//www.bbrc.in/

Doi: http://dx.doi.org/10.21786/bbrc/13.11/20 in an unmanned way. The main objective is to develop an android application which acquires image, identifies the disease type, and suggests the fertilizers and pesticides. This increases the crop yield and reduces manpower and labor cost. In India, rice and wheat are the most widely used food crops. The demand for rice and wheat increases day-by-day. The main obstacle for achieving the target is the bacterial and fungal diseases. In severe cases, these diseases can lead to yield loss up to 50\%. This paper considers three major bacterial and fungal diseases affecting mostly rice and wheat. These diseases are also present in maize, sugarcane, palm, ornamental plants etc.

The two diseases of the rice plant; Leaf Blast and Brown Spot are classified using Self Organizing Map (SOM), an unsupervised learning technique with an accuracy of approximately 92\% (Phadikar and Sil,2008). The authors used multiclass Support Vector Machine (SVM) with Gaussian kernel function for classifying three types of diseases in paddy (Shah, Prajapati and Dabhi, 2016). Fuzzy classifier is used for classifying four different diseases in wheat plant. The accuracy for disease detection and disease type classification are $88 \%$ and 56\% respectively (Diptesh et al., 2014). Artificial

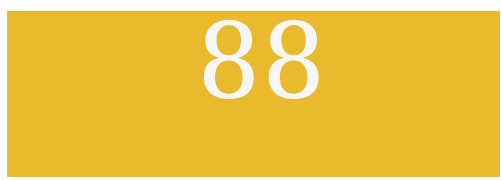


Neural Network (ANN) and SVM classifiers are used for detection and classification of maize diseases using python. ANN has resulted an accuracy of 55\% to 65\% and SVM has resulted an accuracy of 70\% to 75\% (Durga and Anuradha, 2019).Fuzzy expert system is designed and developed for identifying nine different diseases of finger millets (Roseline, Tauro and Ganesan, 2012). The system identifies the diseases by analysing the symptoms for each disease and provides tips to overcome the diseases.

An approach for identifying a pearl millet disease was introduced using Convolutional Neural Network (CNN) model VGG16 and obtained an accuracy of 95\% (Coulibaly et al., 2019). Alexnet is used for identifying 14 different diseases of olive plant for which $80 \%$ of the dataset for training and the remaining $20 \%$ for testing is utilized and obtained an accuracy of $99.11 \%$ (Alruwaili et al., 2019). A system for detecting diseases in palm leaves is developed using multiclass SVM classifier. The system detects two diseases: Anthracnose and Chimaera with an accuracy of 96\% (Masazhar and Kamal, 2018). A system is proposed where four different diseases of groundnut leaves are detected and classified using Back propagation network in MATLAB and obtained an accuracy of 97.41\% (Ramakrishnan and Sahaya, 2015).

Algorithms like Multiclass SVM, Naïve Bayes, K-Nearest Neighbour and Multinomial Logistic Regression were used to classify three different diseases of sunflower with an accuracy of 92.15\%, 89.075\%, 89.32\% and 92.57\% respectively. The accuracy of classifying the healthy leaves are 100\% in all the four classification algorithms (Pinto et al., 2016). Healthy leaves and scorch leaves are classified using K-Nearest Neighbour algorithm with $\mathrm{K}=1$ in MATLAB and obtained an accuracy of about 95\% (Eaganathan et al., 2014). An automated system device is designed to detect Mildew disease from healthy plants and to estimate the severity of the disease. K-Nearest Neighbour algorithm is used for detecting the disease and used decision tree for severity estimation. The accuracy of the experiment is 82.5\% (Parikh et al., 2016). Based on the different parts of plant, 18 classes of diseases were trained and tested using Deep Convolutional Neural Network (DCNN).

Six models were designed and out of which, the performance of ResNet50 and InceptionV2 based models were better. The accuracy of the developed system is about 90\% (Selvaraj et al., 2019). An image processing system is developed using SVM to identify two major diseases of tea namely Algal and Brown Blight from the healthy tea leaves (Hossain et al., 2018). The success rate of the proposed system was about 93\% in which 50 black coffee beans and 50 healthy coffee beans were used. They used grey scale image for colour feature extraction and $\mathrm{K}$-means clustering for segmentation process. The results obtained are 100\% accurate using MATLAB software (Shetty, Kotian and Uppar, 2019). An application which classifies two diseases- coffee leaf miner and coffee leaf rust from the normal coffee leaves is developed using Back propagation algorithm.
The application also estimates the severity of disease (Manso et al., 2019). Texture Based Disease Recognition experiment is conducted and calculated kappa coefficient and sensitivity as 0.900 and 0.933 , respectively. Similarly, they calculated the same parameters using Deep Learning Disease Recognition and obtained the result as 0.970 and 0.980 respectively (Boa Sorte et al., 2019).

The system classifies two cucumber crop diseases namely powdery mildew, downy mildew from the healthy using Artificial Neural Network with the classification accuracy of 80.4\% (Pooja Pawar et al, 2016).Graphical User Interface (GUI) is also developed to indicate the diagnosis and treatment for the detected disease (Pawar, Turkar and Patil, 2016). Backpropagation neural network is used for classifying healthy pomegranate leaves from the diseased leaves and obtained an accuracy of 90\% (Pawar and Jadhav, 2018). The leaves of 3 commonly attacked disease of grape are experimented using MATLAB. The feature extraction using LAB color model given an accuracy of 82.5\% and the feature extraction using both LAB and HSI color model gives an accuracy of 90\%. Multiclass SVM is used for classification of diseases (Agrawal, Singhai and Agarwal, 2018). Classification of two grape diseases downy and powderly was done using SVM classifier with 88.89\% accuracy (Padol and Yadav, 2016). This paper focuses on three main diseases that affects different plants namely - Bacterial leaf streak, Brown spot and Black horse riding.

Bacterial Leaf Streak: Bacterial leaf streak is one of the bacterial diseases which attacks mostly wheat plant. Symptoms initially appear as water-soaked streaks and turns into brown linear lesions between leaf veins. Initially, these leaf streaks are dark green in color and when the disease becomes severe, it turns to brownish or yellowish gray. The complete leaves may become brown and dead when the disease is extremely severe. Applying copper-based fungicide on leaves or pesticide named Epsom salt can help in controlling the disease. The yield loss due to bacterial leaf streak disease is about $10 \%$ to $40 \%$. Bacterial leaf streak disease is shown in figure 1 .

Figure 1: Bacterial leaf streak disease

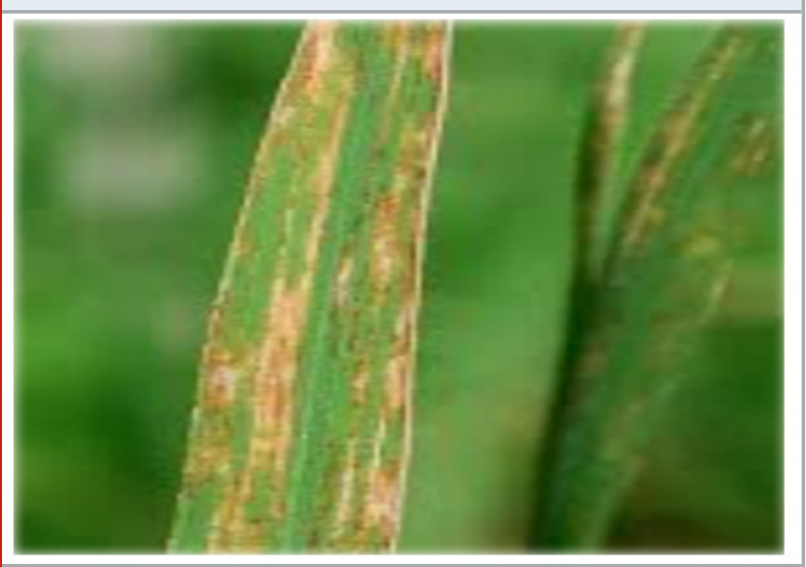

Brown Spot: Brown spot, a fungal disease occurs mostly in rice and soya bean plants. Infected seedlings have 
brown lesion, and the leaves turn to rusty brown. The diseased seedlings die at the early stage or become underdeveloped. Fungicides like triadimefon can help in the reduction of this disease. Leaf wetness can increase the severity of the disease within 6 to 36 hours. The yield loss due to brown spot disease is about $8 \%$ to $15 \%$ but premature defoliation of canopy may increase the yield loss of $25 \%$ to $50 \%$. Brown spot disease is shown in figure 2 .

Figure 2: Brown spot disease

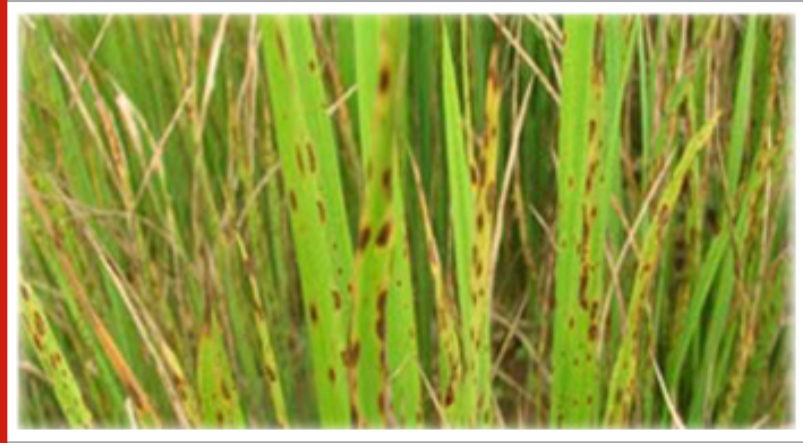

Black Horse Riding: Black horse-riding disease mostly attacks the monocotyledons. Most of the patches caused by this disease are due to pathogenic fungi. Once the fungi enter the leaf, it continues to grow and destroys the leaf tissue. This results in spots of varying sizes. The dead areas on the leaves will be dark in color. The border of the malignant areas in the leaf will be in red or purple. When the disease becomes severe, it leads to complete defoliation of leaves. A chemical fungicide or any number of organic options such as Copper, Lime Sulphur, Neem Oil, Potassium or Ammonium Bicarbonate can be used as fertilizer. Black horse-riding disease is shown in figure 3.

Figure 3: Black horse riding disease

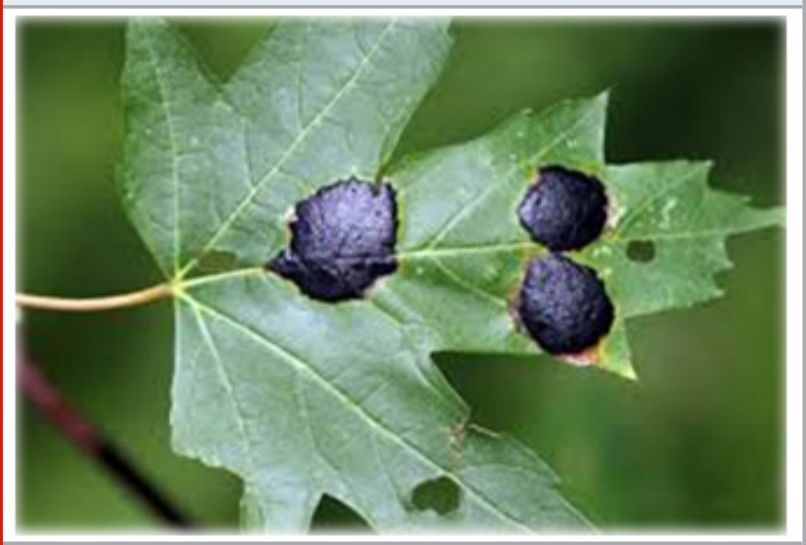

MATERIAL AND METHODS

An android application is developed using Android Studio through which the images are acquired, preprocessed, segmented and classified based on the disease.
Figure 4 represents the flow of the proposed system for plant disease classification.

Figure 4: Proposed system for plant disease classification

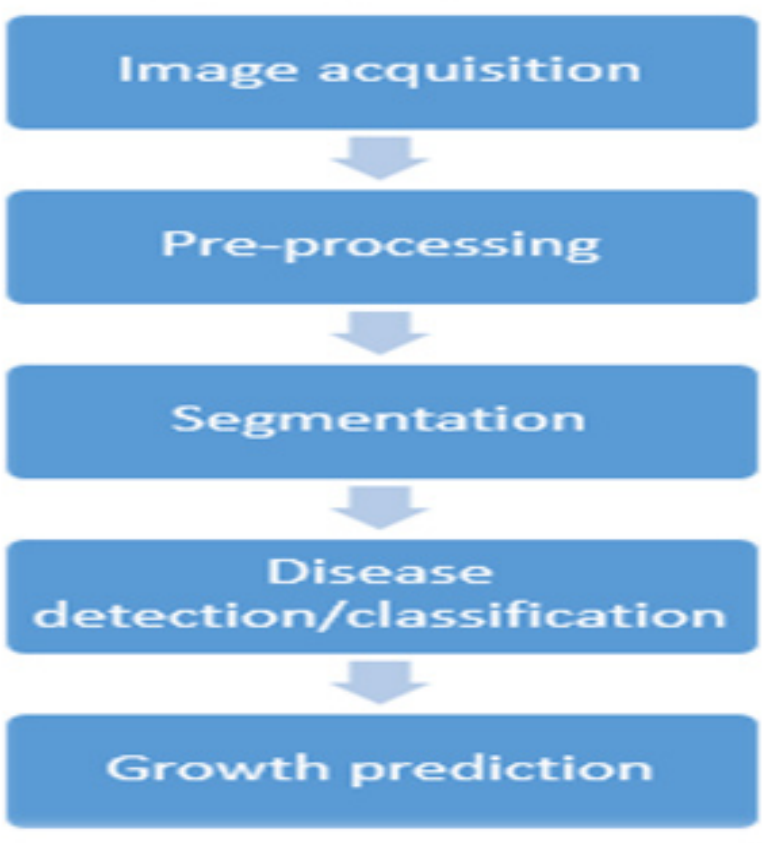

Image Acquisition: In image acquisition, the colored image of the diseased or healthy leaf to be experimented is acquired through high clarity mobile phone or from the internet. The leaves with different canopy size were selected to decrease the difficulty of the classification problem. In the developed Android application, image to be tested is chosen from the gallery as in figure 5 .

Figure 5: Selected Image for testing

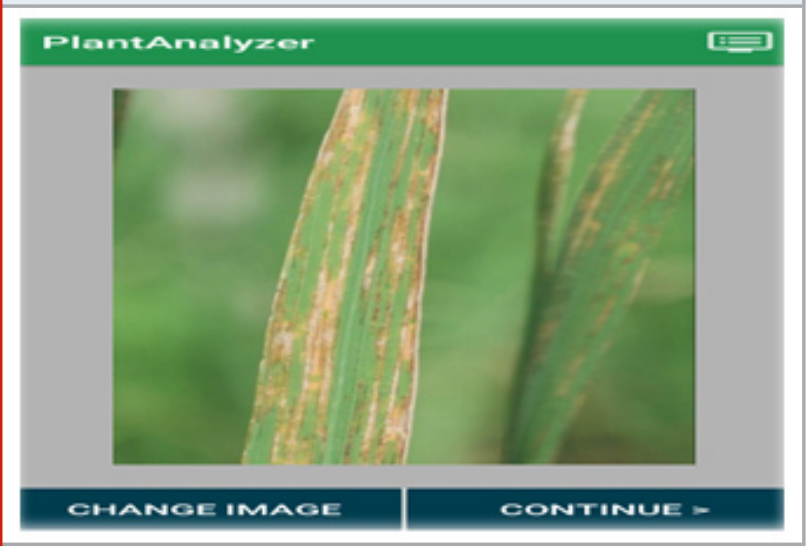

Preprocessing: Image pre-processing is a technique wherein the unwanted noises present in the images are removed. In the proposed work, there are three steps in image pre-processing - RGB to Gray color transformation, Bitmap extraction and Histogram analysis. 
RGB to Gray color transformation: The first step in pre-processing is to convert the colored image of leaf into gray color. RGB images will have three different intensities for each pixel. So, on converting it to gray color, there will be only single intensity for each pixel.

Bitmap Extraction: The second step in pre-processing is the extraction of bitmap so that the required part of the leaf is separated from the background.

Histogram Analysis: The image thus obtained is analyzed with the pixel intensity in order to form the histogram graph. In the histogram graph, the left most area of the horizontal axis denotes the dark tone of the image and similarly the right side denotes the light tone of the image.

Segmentation: Image segmentation is the process of fragmenting an image into several segments. This is done to make the analysis easier. After the histogram analysis, threshold-based segmentation is done. The output of this step will be a binary image. This is the simplest method used for image segmentation, but it is still effective. The segmented image is shown in figure 6 .

Figure 6: Image segmentation

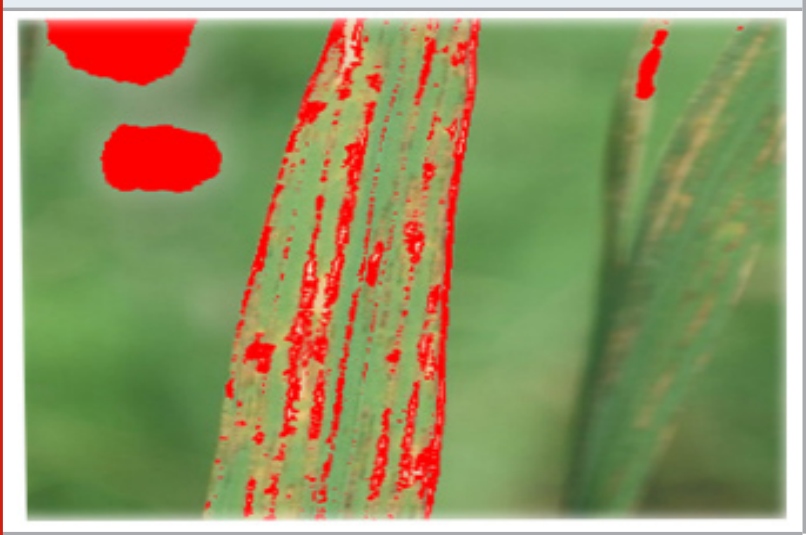

Disease detection and classification: After the segmentation process, images are classified based on Naive Bayes algorithm. This algorithm obeys Bayes theorem. It comes under the category of probabilistic classifiers. It needs only a smaller number of data sets compared to other classifiers. There will be different pixel values for each disease. The input image to be tested compares with all possible diseases after the segmentation process. It predicts the probability for all the possible diseases and the output will be the disease which has the highest probability. The application also displays the percentage of the diseased portion.

Growth prediction: Based on the size and shape of the leaf, its growth is predicted. The application also predicts the sunlight condition if it is good or not. If the percentage of disease is more than 5\%, then the sunlight condition is not good. Based on the disease type, the android application suggests the fertilizers and pesticides.
Figure 7: Input image to be tested

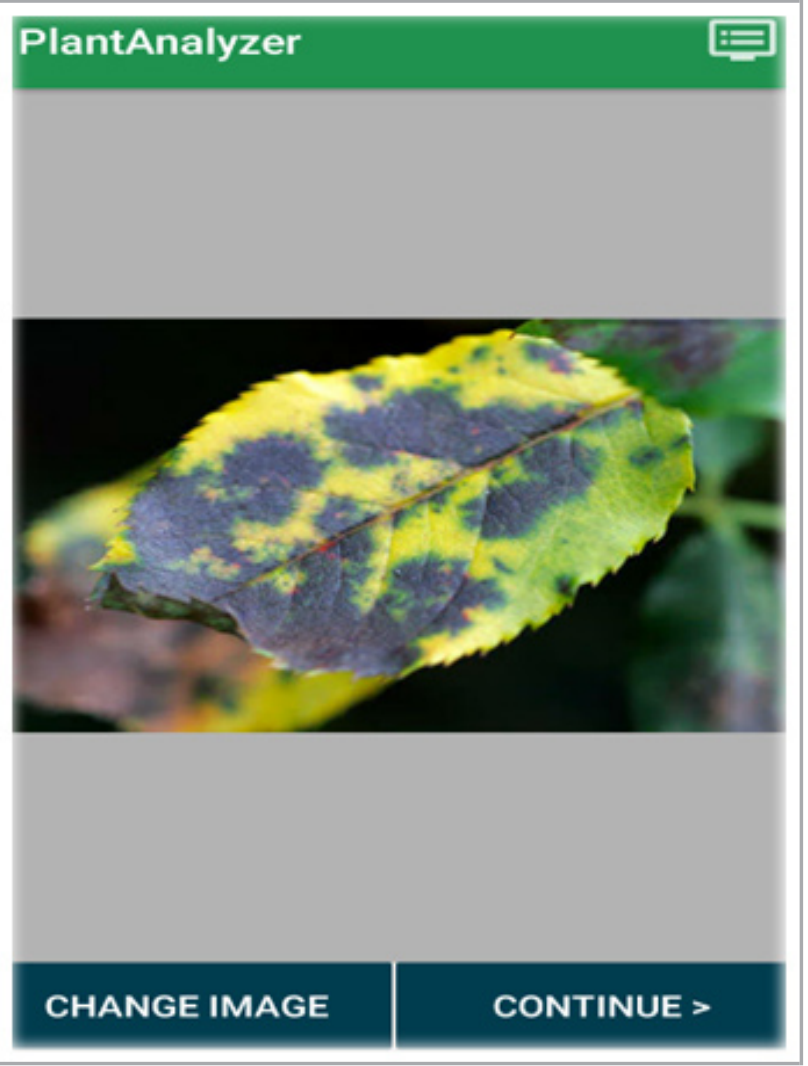

\section{RESULTS AND DISCUSSION}

The android application developed is installed in the android device. The image to be tested is selected from the gallery. The app has two options - one is to change the image and the other is to continue. These two choices are fixed using the Android studio press. The application screen of the same is shown in figure 7.

Once continue is pressed, it will move to the next page of the application. In this step, the given input image checks with all three diseases Bacterial leaf streak, brown spot, and black horse riding. The portion matching with the diseases will be red in color as in figure 8. If report option is pressed, then the disease that has the maximum probability will be displayed along with the percentage of infection as shown in figure 9. A button is inserted in the bottom of this page. Once this button is pressed, the application will predict the sunlight condition if it is good or not and predicts the growth of the plant. It also suggests the fertilizers and pesticides based on the type of the disease as shown in figure 10. A button is inserted in that page for sharing these suggestions.

For each disease, five images were tested. For bacterial leaf streak, all the five images were classified correctly and its accuracy is $100 \%$. In case of brown spot, three images were identified correctly out of five images with the accuracy 60\% and for Black horse riding disease, four images were identified correctly with the accuracy of $80 \%$.The average accuracy obtained is $80 \%$.The graphical 
representation of the classified image accuracy is shown in Figure 11. Here, the $\mathrm{x}$-axis denotes the disease type and the $y$-axis denotes the accuracy.

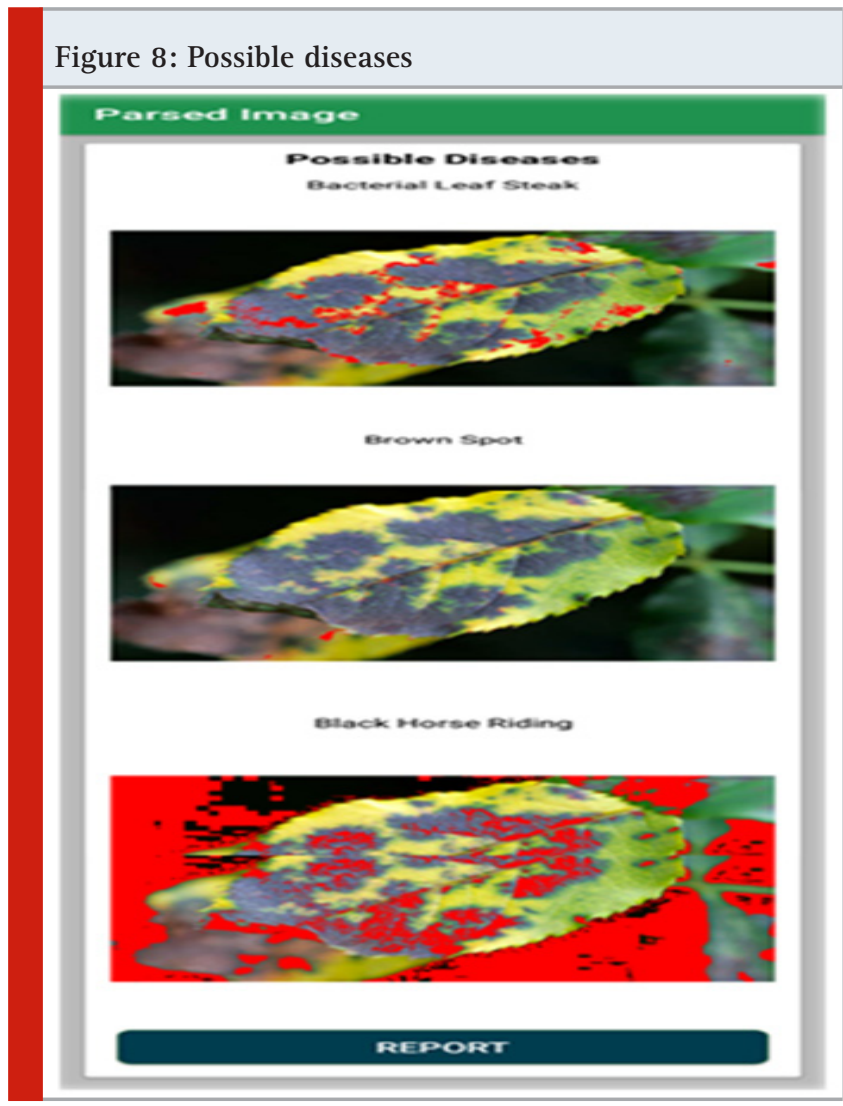

Figure 10: Fertilizers and pesticides suggestion

\begin{tabular}{|c|}
\hline Fertilizers \\
\hline Fertilizer \\
\hline Disease: Black Horse Riding \\
\hline Sun Light Condition: NOT GOOD \\
\hline Growth Prediction: 5 Months (Not Good) \\
\hline $\begin{array}{l}\text { Fertilizer: You can use a chemical fungicide } \\
\text { or any number of organic options such as: } \\
\text { Copper, Lime Sulfur, Neem Oil, Potassium or } \\
\text { Ammonium Bicarbonate, Sulfur. }\end{array}$ \\
\hline
\end{tabular}

Pesticide: Essential Oil Blends

Essential oils can be put to great use in the garden. They work to do everything from attracting pollinators to suppressing fungus.

Mix a teaspoon of vodka with 10 drops of lemon essential oil, 10 drops of eucalyptus oil, 10 drops of cedarwood oil and an ounce of water. Add to a glass spray bottle ang apply thoroughly, shaking before each
Figure 9: Disease type

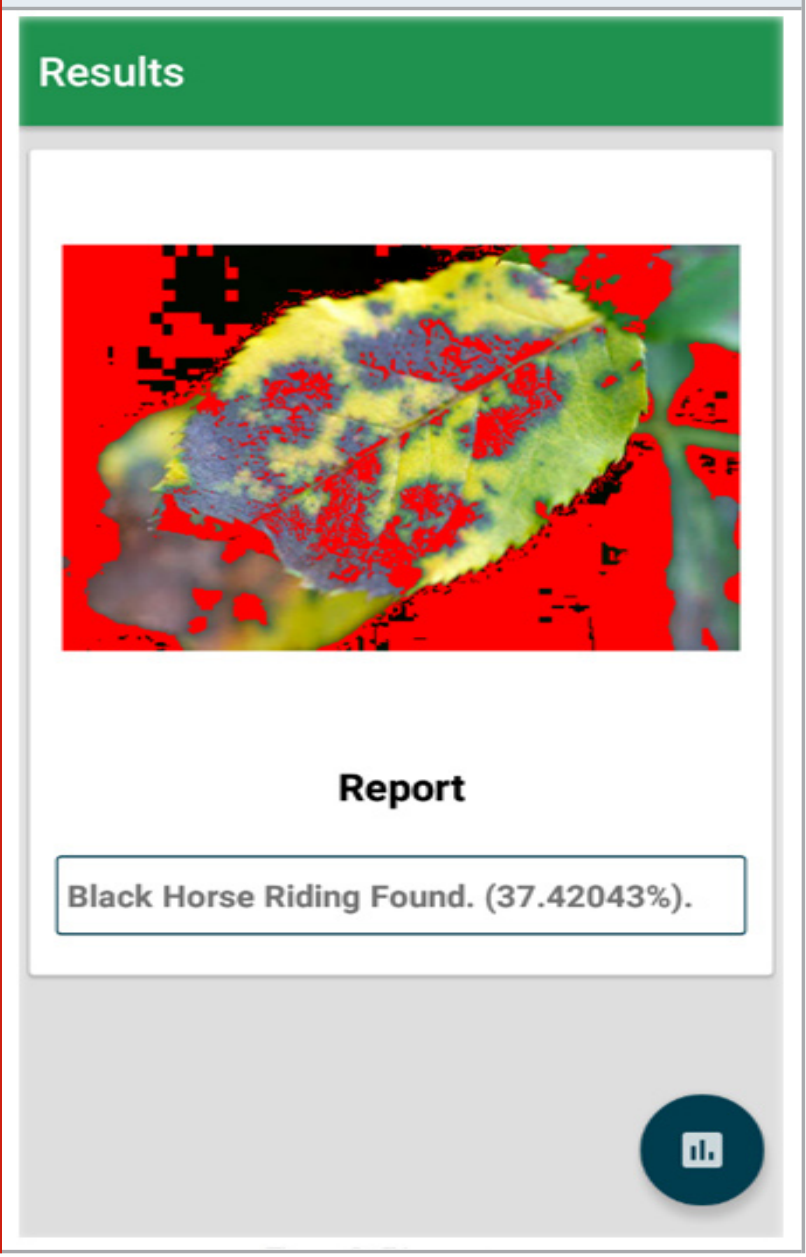

Figure 11: Performance Analysis

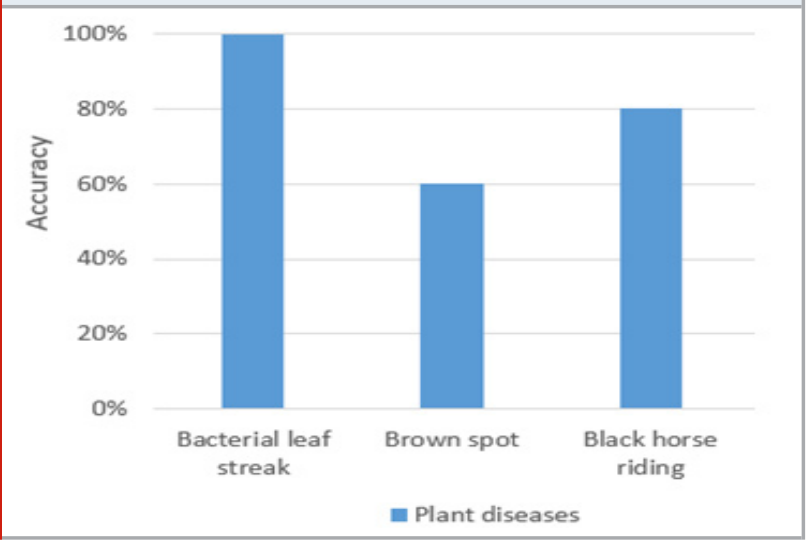

\section{CONCLUSION}

The developed android application uses RGB to gray color transformation, bitmap extraction and histogram analysis for image pre-processing. Thresholding based segmentation is done and finally disease type is classified using Naïve Bayes classifier. In this experiment, three class of plant diseases - Bacterial leaf streak, Brown spot and Black horse riding are considered. For testing 
purpose, 15 images are used. The average accuracy of the application is $80 \%$. In future, other algorithms like decision tree can be applied to improve the accuracy.

\section{ACKNOWLEDGEMENTS}

Nil

Conflict of interest: The author declares that there is no conflict of interest.

\section{REFERENCES}

Agrawal, N., Singhai, J. and Agarwal, D. K. (2018) Grape leaf disease detection and classification using multi-class support vector machine, International Conference on Recent Innovations in Signal Processing and Embedded Systems, RISE 2017. IEEE, 2018-Janua, pp. 238-244. doi: 10.1109/RISE.2017.8378160.

Alruwaili, M. et al. (2019) An efficient deep learning model for olive diseases detection, International Journal of Advanced Computer Science and Applications, 10(8), pp. 486-492. doi: 10.14569/ijacsa.2019.0100863.

Boa Sorte, L. X. et al. (2019) Coffee Leaf Disease Recognition Based on Deep Learning and Texture Attributes, Procedia Computer Science, 159, pp. 135144. doi: 10.1016/j.procs.2019.09.168.

Coulibaly, S. et al. (2019) Deep neural networks with transfer learning in millet crop images, Computers in Industry, 108, pp. 115-120. doi: 10.1016/j. compind.2019.02.003.

Diptesh, M. et al. (2014) Application of Fuzzy C-Means Clustering Method to Classify Wheat Leaf Images based on the presence of rust disease, Advances in Intelligent Systems and Computing, 327(October). doi: 10.1007/978-3-319-11933-5.

Durga, N. K. and Anuradha, G. (2019) Plant disease identification using SVM and ANN algorithms, International Journal of Recent Technology and Engineering, 7(5), pp. 471-473.

Eaganathan, U. et al. (2014) Identification of Sugarcane Leaf Scorch Diseases using K-means Clustering Segmentation and K-NN based Classification, International Journal of Advances in Computer Science and Technology, 3(12), pp. 11-16.

Hossain, M. S. et al. (2018) Recognition and detection of tea leaf's diseases using support vector machine, Proceedings - 2018 IEEE 14th International Colloquium on Signal Processing and its Application, CSPA 2018, (March), pp. 150-154. doi: 10.1109/ CSPA.2018.8368703.

Manso, G. L. et al. (2019) A smartphone application to detection and classification of coffee leaf miner and coffee leaf rust. Available at: http://arxiv.org/ abs/1904.00742.

Masazhar, A. N. I. and Kamal, M. M. (2018) Digital image processing technique for palm oil leaf disease detection using multiclass SVM classifier, 2017 IEEE International Conference on Smart Instrumentation, Measurement and Applications, ICSIMA 2017, 2017-November, pp. 1-6. doi: 10.1109/ICSIMA.2017.8311978.

Padol, P. B. and Yadav, A. A. (2016) SVM classifier based grape leaf disease detection, Conference on Advances in Signal Processing, CASP 2016, pp. 175-179. doi: 10.1109/CASP.2016.7746160.

Parikh, A. et al. (2016) Disease detection and severity estimation in cotton plant from unconstrained images, Proceedings - 3rd IEEE International Conference on Data Science and Advanced Analytics, DSAA 2016, pp. 594-601. doi: 10.1109/DSAA.2016.81.

Pawar, P., Turkar, V. and Patil, P. (2016) Cucumber disease detection using artificial neural network, Proceedings of the International Conference on Inventive Computation Technologies, ICICT 2016, 2016. doi: 10.1109/INVENTIVE.2016.7830151.

Pawar, R. and Jadhav, A. (2018) Pomogranite disease detection and classification, IEEE International Conference on Power, Control, Signals and Instrumentation Engineering, ICPCSI 2017. IEEE, pp. 2475-2479. doi: 10.1109/ICPCSI.2017.8392162.

Phadikar, S. and Sil, J. (2008) Rice disease identification using pattern recognition techniques, Proceedings of 11th International Conference on Computer and Information Technology, pp. 420-423. doi: 10.1109/ ICCITECHN.2008.4803079.

Pinto, L. S. et al. (2016) Crop Disease Classification using Texture Analysis,IEEE International Conference on Recent Trends in Electronics Information Communication Technology, pp. 825-828.

Ramakrishnan, M. and Sahaya, A. N. A. (2015) Groundnut leaf disease detection and classification by using back probagation algorithm, 2015 International Conference on Communication and Signal Processing, ICCSP 2015, (7092512506), pp. 964-968. doi: 10.1109/ ICCSP.2015.7322641.

Roseline, P., J.M Tauro, C. and Ganesan, N. (2012) Design and Development of Fuzzy Expert System for Integrated Disease Management in Finger Millets, International Journal of Computer Applications, 56(1), pp. 31-36. doi: 10.5120/8857-2815.

Selvaraj, M. G. et al. (2019) AI-powered banana diseases and pest detection, Plant Methods. BioMed Central, 15(1), pp. 1-11. doi: 10.1186/s13007-019-0475-Z.

Shah, J. P., Prajapati, H. B. and Dabhi, V. K. (2016) A survey on detection and classification of rice plant diseases, IEEE International Conference on Current Trends in Advanced Computing (February 2019). doi: 10.1109/ICCTAC.2016.7567333.

Shetty, B., Kotian, K. R. and Uppar, A. S. (2019) Coffee Bean Detection and Segregation, International Journal of Engineering Science and Computing, 9(4), pp. 21636-21638. 